\title{
Prostaglandin Reductase 1 as a Potential Therapeutic Target for Cancer Therapy
}

\author{
Xing Wang ${ }^{1,2}$, Guobing Yin ${ }^{1 *}$, Wei Zhang ${ }^{2}$, Kunlin Song ${ }^{2}$, Longbin Zhang ${ }^{2}$ and Zufeng Guo ${ }^{2 *}$ \\ ${ }^{1}$ Department of Breast and Thyroid Surgery, Second Affiliated Hospital of Chongqing Medical University, Chongqing, China, \\ ${ }^{2}$ Center for Novel Target and Therapeutic Intervention, Institute of Life Sciences, Chongqing Medical University, Chongqing, China
}

OPEN ACCESS

Edited by:

Dipak Panigrahy,

Harvard Medical School,

United States

Reviewed by:

Nune Markosyan,

University of Pennsylvania,

United States

Marianela Vara Messler,

Catholic University of Louvain,

Belgium

*Correspondence:

Guobing Yin

yinguobing@cqmu.edu.cn

Zufeng Guo

guozufeng@hotmail.com

Specialty section:

This article was submitted to Inflammation Pharmacology,

a section of the journal

Frontiers in Pharmacology

Received: 31 May 2021

Accepted: 29 July 2021

Published: 06 August 2021

Citation:

Wang $X$, Yin G, Zhang W, Song $K$,

Zhang $L$ and Guo $Z$ (2021)

Prostaglandin Reductase 1 as a

Potential Therapeutic Target for

Cancer Therapy.

Front. Pharmacol. 12:717730.

doi: 10.3389/fphar.2021.717730
Altered tumor metabolism is a hallmark of cancer and targeting tumor metabolism has been considered as an attractive strategy for cancer therapy. Prostaglandin Reductase 1 (PTGR1) is a rate-limiting enzyme involved in the arachidonic acid metabolism pathway and mainly responsible for the deactivation of some eicosanoids, including prostaglandins and leukotriene B4. A growing evidence suggested that PTGR1 plays a significant role in cancer and has emerged as a novel target for cancer therapeutics. In this review, we summarize the progress made in recent years toward the understanding of PTGR1 function and structure, highlight the roles of PTGR1 in cancer, and describe potential inhibitors of PTGR1. Finally, we provide some thoughts on future directions that might facilitate the PTGR1 research and therapeutics development.

Keywords: PTGR1, tumor metabolism, eicosanoid pathways, cancer therapeutics, inhibitor

\section{INTRODUCTION}

Cancer is a major global burden of disease and the second leading cause of death over a period of decades (Siegel et al., 2021; Sung et al., 2021). In recent years, cancer treatment and diagnosis have made remarkable progress, especially in immunotherapy (Hiam-Galvez et al., 2021; Salas-Benito et al., 2021), targeted therapies (Oh and Bang, 2020), molecular diagnostics (Sokolenko and Imyanitov, 2018), screening and early detection (Loud and Murphy, 2017) and so on. Although many cancers have become the chronic and even curable disease, tumor heterogeneity and acquired drug resistance remain two of the biggest challenges for cancer therapy, highlighting needs of novel anti-cancer strategies. Recently, an increasingly large body of evidence indicates that metabolic reprogramming, recognized as one of the cancer hallmarks (Hanahan and Weinberg, 2011), plays an essential role in cancer and targeting metabolic reprogramming has ability to develop novel strategies for cancer therapy (Fendt et al., 2020).

Arachidonic acid (AA) is a polyunsaturated fatty acid present in the phospholipids of cell membranes and can be metabolized through cyclooxygenase (COX), lipoxygenase (LOX) and P450 epoxygenase pathways to generate eicosanoids, including prostanoids (PGs), leukotrienes, hydroxyeicosatetraenoic acids and so on (Wang et al., 2021). Prostaglandin Reductase 1 (PTGR1) belongs to the medium-chain dehydrogenase/reductase superfamily and it is involved in both COX and LOX downstream pathways to further metabolize eicosanoids (Figure 1). The altered metabolism of AA has long been shown to have crucial roles in cancer progression. For example, COX-2 expression is upregulated in multiple cancers, including colorectal, stomach, liver, lung and bladder, and increased COX-2 expression is associated with poor clinical outcomes. In cancer, the most abundant COX-2 product is prostaglandin $\mathrm{E}_{2}\left(\mathrm{PGE}_{2}\right)$ which has a predominant role in promoting tumor growth and is also associate with a poor prognosis (Wang and Dubois, 2010). LOX-derived leukotriene B4 (LTB4), 


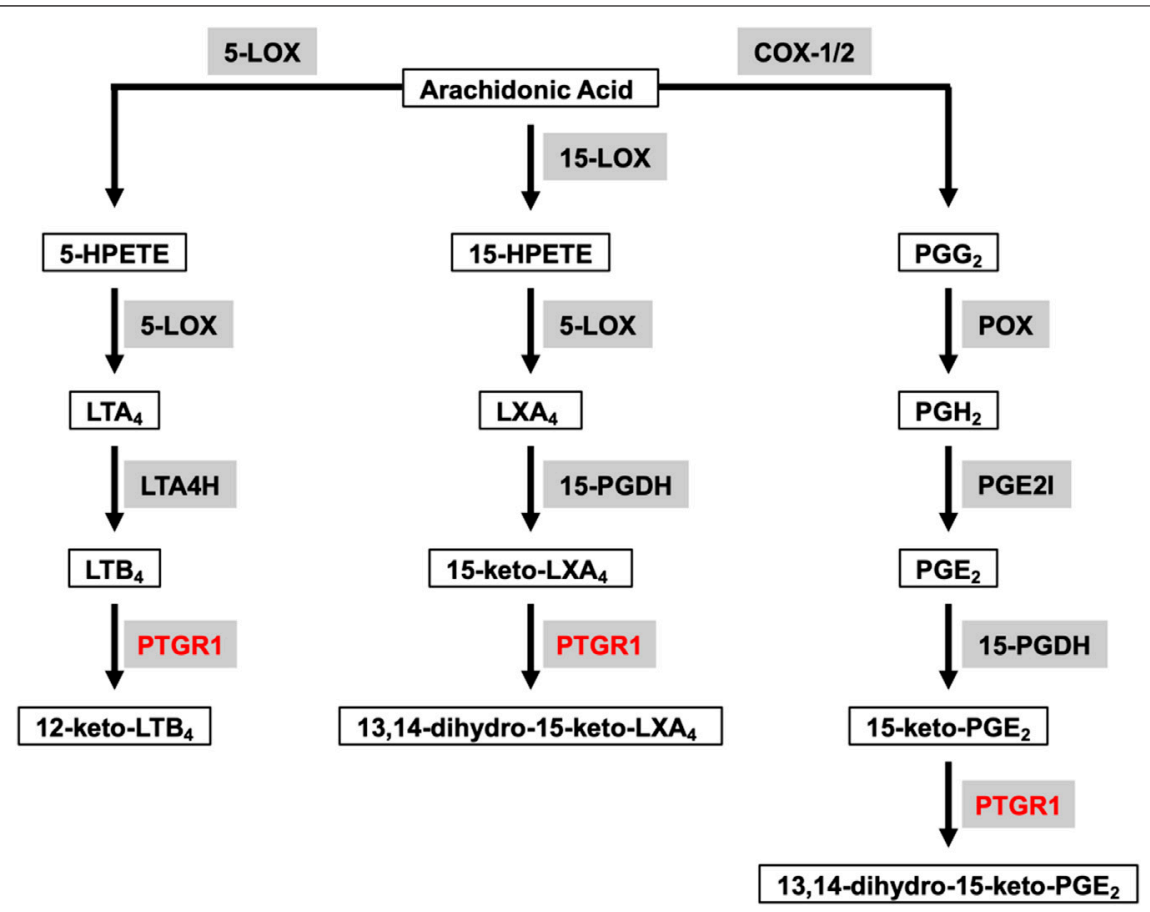

FIGURE 1 | Overview of PTGR1 function and its endogenous substrates. Arachidonic Acid can be metabolized through COX and LOX pathways to generate eicosanoids, such as prostaglandins, leukotrienes and lipoxins. $\mathrm{LTB}_{4}, 15-\mathrm{k}$-to--LXA 4 , and 15-keto-PGE 2 are the direct substrates for PTGR1. Substrates are emphasized with black box. Enzymes are emphasized in grey background and PTGR1 are colored with red. 5-LOX, 5-lipoxygenase; 15-LOX, 15-lipoxygenase; COX-1, cyclooxygenase-1; COX-2, cyclooxygenase-2; 5-HPETE, 5-hydroperoxyeicosatetraenoic acid; 15-HPETE, 15-hydroperoxyeicosatetraenoic acid; PGG $_{2}$, PGH ${ }_{2}$, and $P G E_{2}$ are prostaglandins $G 2, H 2$ and $E 2$, respectively; $L T A_{4}$ and $L T B_{4}$ are leukotrienes $A 4$ and $B 4$, respectively; $L X A_{4}$, lipoxin $A 4$; $L T A 4 H$, leukotriene $A 4$ hydrolase; 15-PGDH, 15-hydroxyprostaglandin dehydrogenase; PGE2I, prostaglandin E2 isomerase.

through stimulating its receptor BLT1, has also been shown to promote TGF- $\beta$-mediated proliferation in breast cancer cells (Jeon et al., 2015).

Recently, a growing evidence suggested that PTGR1 plays a significant role in cancer progression (Xue et al., 2016), cancer prognosis (Györffy et al., 2013), chemotherapeutic sensitivity (Dick et al., 2004) and cancer oxidative stress (SánchezRodríguez et al., 2017). Therefore, PTGR1 is a novel potential therapeutical target for cancer treatment.

\section{The Function and Structure of PTGR1}

PTGR1 is a NADPH-dependent alkenal/one oxidoreductase (AOR), which catalyzes the reduction of double bond of $\alpha / \beta$-unsaturated ketones, alkenals and nitroalkenes. PTGR1 has broad spectrum of endogenous substrates and is involved in the degradation of PGs, $\mathrm{LTB}_{4}$, lipoxins and so on (Figure 1). $\mathrm{LTB}_{4}$ is one of the direct substrates of PTGR1 and can be oxidized to 12-keto- $\mathrm{LTB}_{4}$, which is approximately 100 times less potent than $\mathrm{LTB}_{4}$ (Yokomizo et al., 1993). However, PGs (such as $\mathrm{PGE}_{2}, \mathrm{PGJ}_{2}$, and $\mathrm{PGF}_{2 \alpha}$ ) are converted into 15-ketoPGs first by NAD-dependent 15-hydroxyprostaglandin dehydrogenase (15-PGDH), and then further metabolized into biologically less active 13,14-dihydro-15-keto-PGs by PTGR1. Similarly, $\mathrm{LXA}_{4}$ is oxidized to 15 -keto-LXA $\mathrm{LA}_{4}$ byPGDH and subsequently converted to 13,14-dihydro-15-ketoLXA $_{4}$ by PTGR1 (Clish et al., 2000). In comparison to 15-keto-
$\mathrm{PGE}_{1}$, 15-keto-PGF $1 \alpha$, 15-keto-PGF $2 \alpha$, and $\mathrm{LTB}_{4}$, 15-keto$\mathrm{PGE}_{2}$ is the best substrate for PTGR1 with the highest $k \mathrm{cat} /$ $K \mathrm{~m}$ value. For example, the $k \mathrm{cat} / \mathrm{Km}$ value for 15 -keto- $\mathrm{PGE}_{2}$ reduction is approximately 200 times higher than that for $\mathrm{LTB}_{4}$ oxidation (Mesa et al., 2015). Nitro oleic acid (NO-OA), another substrate of PTGR1, is an endogenous electrophilic compound with the capacity to modify thiols in proteins. PTGR1 is able to deactivate $\mathrm{NO}_{2}-\mathrm{OA}$ to non-electrophilic product $\mathrm{NO}_{2}$-SA through reduction (Vitturi et al., 2013). In addition to degradation of endogenous substrates, PTGR1 has ability to activate some drugs with $\alpha / \beta$-unsaturated ketone. For example, CS-670 (Itoh et al., 2008), non-steroidal antiinflammatory drugs (NSAIDs), and irofulven (hydroxymethylacylfulvene, HMAF) (McMorris et al., 1999) are converted into the active electrophilic metabolites by PTGR1 and then play their pharmacological role in vitro and in vivo.

There are only two structures of PTGR1 have been reported so far. In 2006, Hori et al. solved the crystal structure of guinea-pig PTGR1 in ternary complex with $\mathrm{NADP}^{+}$and indomethacin and revealed that the broad spectrum of indomethacin efficacy can be attributed to its ability to adopt a range of different stable conformations with target proteins, including PTGR1 (Hori et al., 2006). In 2010, Yue et al. deposited the other unpublished structure of hPTGR1 in complex with $\mathrm{NADP}^{+}$ and raloxifene into Protein Data Bank (PDB) (Yue et al., 


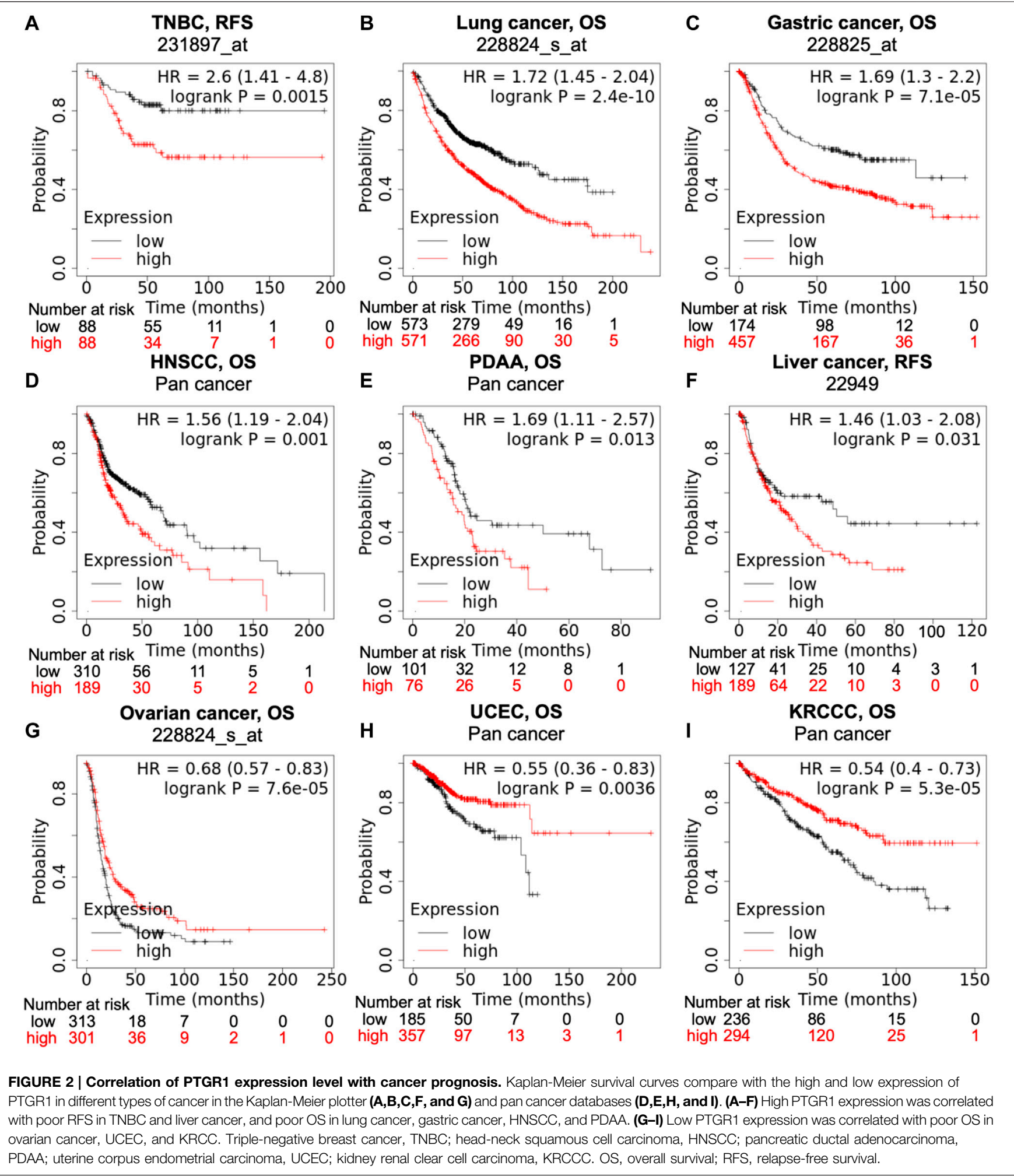

2011). Based on the hPTGR1 structure, Mesa et al. applied molecular docking simulations and site-directed mutagenesis assays to indicate that Arg56 plays an important role in binding the $\alpha$-chain carboxylic group of 15 -keto-PGE $\mathrm{PG}_{2}$ and
15-d-PGJ ${ }_{2}$ through an ionic interaction (Mesa et al., 2015). In addition, hPTGR1 inhibitors, niflumic acid and indomethacin have been found to establish ionic interactions with Arg56 through their carboxylic acid group, suggesting that the 
carboxylic acid stands as a good candidate pharmacophore group in the search of novel PTGR1 inhibitors (Mesa et al., 2015).

\section{PTGR1 and Cancer Progression}

Overexpressed PTGR1 was found in many cancer cell lines, such as hepatocellular carcinoma (Sánchez-Rodríguez et al., 2017), lung cancer (Zhao et al., 2010), prostate cancer (Xue et al., 2016), and bladder cancer (Tapak et al., 2015), suggesting that PTGR1 has an oncogenic role. In addition, knockdown of PTGR1 was found to inhibit cell proliferation of multiple cancer cell lines. For example, Liu et al. found that knockdown of PTGR1 not only decreased the proportion of breast cancer stem cells, but also inhibited cell proliferation of TNBC cells (Liu et al., 2018). Similarly, Roberts et al. reported that knockdown of PTGR1 or inhibition of PTGR1 by its inhibitor was able to suppress TNBC cell growth (Roberts et al., 2017). In 2016, Xue et al. also demonstrated that knockdown of PTGR1 slowed down prostate cancer cell proliferation by inducing cell cycle arrest and apoptosis (Xue et al., 2016). In PC3 cells, PTGR1 silencing was found to increase the expression of key cell cycle inhibitor P21, cleaved-PARP and caspase 3, and decrease the expression of cyclin D1 (Xue et al., 2016). Together, these studies suggest that PTGR1 plays a role in cancer cell proliferation and can be of interest as a potential target for cancer therapy.

\section{PTGR1 and Cancer Prognosis}

Data from the KM-plotter and Pan-cancer database suggests that high PTGR1 expression correlates with poor prognosis in a variety of tumor types, such as triple-negative breast cancer (TNBC), lung, gastric, head and neck, pancreatic and liver cancer (Figures 2A-F). On the contrary, in ovarian cancer, uterine corpus endometrial carcinoma, and kidney renal clear cell carcinoma, low PTGR1 level is related with the poor prognosis (Figures 2G-I). Recently, multiple studies also provided further evidence to support that the expression level of PTGR1 was related with cancer prognosis. First, Tapak et al. reported that the high expression of PTGR1 was associated with a decrease in survival time in bladder cancer (Tapak et al., 2015). Second, in colorectal cancer, Yang et al. found that low PTGR1 expression was associated with poor disease-free survival in all stages (Yang et al., 2020). Last but not least, in gliomasphere model, Laks et al. demonstrated that low expression of PTGR1 was related to the aggressive phenotypes, including faster rate of proliferation, greater sphere total volume and increased sphere formation (Laks et al., 2016). These studies suggest that PTGR1 might be a novel prognostic biomarker in cancer and treatment with PTGR1 inhibitor or activator could benefit certain types of cancer patients.

\section{PTGR1 and Chemotherapeutic Sensitivity}

As mentioned above, PTGR1 has ability to activate some prodrugs with $\alpha / \beta$-unsaturated ketone, including anticancer agent HMAF (Raymond et al., 2004; Gong et al., 2006), suggesting that increasing PTGR1 expression level might enhance the efficacy of these drugs. Indeed, Dick et al. reported that human HEK293 cells with the overexpression of
hPTGR1 were 100-times more sensitive than control cells to HMAF (Dick et al., 2004). They also confirmed that PTGR1 activity was positively correlated with HMAF sensitivity using NCI 60 human tumor cell line panel (Dick et al., 2004). In addition, PTGR1 expression was greatly enhanced by NRF2 inducers, such as D3T (Primiano et al., 1996), resveratrol (Yu et al., 2012) and curcumin (Yu et al., 2012). Yu et al. demonstrated that these NRF2 inducers significantly enhance the sensitivity of $\mathrm{HMAF}$ in colon and liver cancer cell lines from 2 to 10 fold ( $\mathrm{Yu}$ et al., 2012). Therefore, these data indicate that PTGR1 could be used to design diagnostic tools or for combination cancer treatment.

\section{PTGR1 and Cancer Oxidative Stress}

PTGR1 has alkenal/one oxidoreductase activity, suggesting that it might have an antioxidant effect against cancer oxidative stress. By studying PTGR1 expression during liver carcinogenesis, Sanchez-Rodriguez et al. found that PTGR1 stimulates cell proliferation and protected cells against ROS-induced cell death (Sánchez-Rodríguez et al., 2017). This result demonstrates that, indeed, PTGR1 has an antioxidant effect and perturbing the redox balance of tumor cells could be a strategy for inducing cell death. More recently, Hatem et al. discovered that auranofin (AUF) and vitamin C (VC) combinations show a synergistic and $\mathrm{H}_{2} \mathrm{O}_{2}$-mediated cytotoxicity to TNBC cell lines and higher PTGR1 expression are more resistance to AUF/VC combination (Hatem et al., 2019). These results suggest that PTGR1 could be used as an effective biomarker for response of cancer cells to ROS-mediated cancer treatment. In addition, PTGR1 inhibitor might sensitize cancer cells to ROS-induced cell death, which could be used to develop novel combination cancer treatment.

\section{PTGR1 Inhibitors}

Although PTGR1 is a promising therapeutic target for cancer therapy, currently only a few PTGR1 inhibitors have been reported. NSAIDs are well-known inhibitors of COX-1 and COX-2 enzymes and able to reduce PTGR1 main substrate prostaglandins. Interestingly, some NSAIDs were also found to inhibit PTGR1. First, Clish et al. screened a panel of NSAIDs at $100 \mu \mathrm{M}$ and found that indomethacin was the best PTGR1 inhibitor with more than 95\% inhibition, while niflumic acid and diclofenac have about 80 and $70 \%$, respectively, (Clish et al., 2001). Hori et al. also reported that indomethacin was able to inhibit the 15 -keto- $\mathrm{PGE}_{2}$ reductase activity of guinea-pig PTGR1 with an $\mathrm{IC}_{50}$ of $97.9 \mu \mathrm{M}$ (Hori et al., 2006). For hPTGR1, Mesa et al. demonstrated that niflumic acid and indomethacin were the best inhibitors with an $\mathrm{IC}_{50}$ of 7.1 and $8.7 \mu \mathrm{M}$, respectively, (Mesa et al., 2015). More recently, Roberts et al. reported that Licochalcone A blocked the $\mathrm{NADP}^{+}$ binding site of PTGR1 and inhibited cell growth in TNBC cells with an $\mathrm{IC}_{50}$ of $8.4 \mu \mathrm{M}$ (Roberts et al., 2017).

\section{Discussion and Future Directions}

In recent years, a growing body of literature has emphasized that metabolic enzyme PTGR1 plays a significant role in cancer and is 
a novel potential therapeutical target for cancer treatment. Although extensive efforts have been made, our current understanding of PTGR1 still remains at a juvenile stage.

First, no systematical approach was used to investigate the substrate spectrum of PTGR1. Metabolomic analysis on PTGR1 knockout cell lines may be useful to identify novel substates and provide the whole substrate spectrum for PTGR1. Second, most of the molecular understanding of PTGR1 is derived from experiments using docking simulations and site-directed mutagenesis assays. Solving of high-resolution 3D structures of hPTGR1 or complexed with its substrates and/or known inhibitors would further improve our knowledge of PTGR1's function and help future computer-aided inhibitor design as well. Third, both PTGR1 upstream substrate $\mathrm{PGE}_{2}$ and direct substrate $\mathrm{LTB}_{4}$ play important roles in cancer progression through promoting tumor growth (Wang and Dubois, 2010; Jeon et al., 2015). This looks like in contrast with those studies demonstrating that knockdown of PTGR1 was able to inhibit cancer cell proliferation (Xue et al., 2016; Roberts et al., 2017; Liu et al., 2018). Currently limited information is available regarding the role of PTGR1 in cancer progression. One possibility is that PTGR1 might produce some metabolites, which play bigger pro-tumorigenic roles than $\mathrm{PGE}_{2}$ and $\mathrm{LTB}_{4}$ in multiple types of cancer cells, including at least TNBC and prostate cancer. Future studies should investigate the role of PTGR1 metabolites and focus on better understanding the inhibitory mechanisms of PTGR1 in cancer progression. Fourth, the correlation of PTGR1 and cancer survival remains questionable. Multiple studies have demonstrated that knockdown of PTGR1 can inhibit cell proliferation of TNBC cells (Roberts et al., 2017; Liu et al., 2018), which is consistent with the finding that high PTGR1 expression correlates with poor prognosis in TNBC cells. However, comparatively little is known why the correlation of PTGR1 and cancer survival exists in other cancer types and has opposing directions in different cancer types. It is worth noting that PTGR1 can change the sensitivity of some anticancer drugs, such as irofulven (McMorris et al., 1999). The correlation analysis will be affected if patients were

\section{REFERENCES}

Clish, C. B., Levy, B. D., Chiang, N., Tai, H.-H., and Serhan, C. N. (2000). Oxidoreductases in Lipoxin A4 Metabolic Inactivation. J. Biol. Chem. 275, 25372-25380. doi:10.1074/jbc.m002863200

Clish, C. B., Sun, Y.-P., and Serhan, C. N. (2001). Identification of Dual Cyclooxygenase-Eicosanoid Oxidoreductase Inhibitors: NSAIDs That Inhibit PG-LX Reductase/LTB4 Dehydrogenase. Bio. chem. biophysical Res. Commun. 288, 868-874. doi:10.1006/ bbrc. 2001.5841

Dick, R. A., Yu, X., and Kensler, T. W. (2004). NADPH Alkenal/One Oxidoreductase Activity Determines Sensitivity of Cancer Cells to the Chemotherapeutic Alkylating Agent Irofulven. Clin. Cancer Res. 10, 1492-1499. doi:10.1158/1078-0432.ccr-03-0162

Fendt, S.-M., Frezza, C., and Erez, A. (2020). Targeting Metabolic Plasticity and Flexibility Dynamics for Cancer Therapy. Cancer Discov. 10, 1797-1807. doi:10.1158/2159-8290.cd-20-0844 treated with irofulven. Therefore, it is important to know the treatment status of the patients included in the analyses. Today, big data and AI are developing so fast, which is boosting the development of bioinformatic analysis. These methods would provide more evidence to answer whether PTGR1 is a novel biomarker for cancer prognosis. Finally, one of the most challenging yet exciting areas of future research will be the development of novel structure and high activity PTGR1 modulators, including inhibitors and activators. Currently, only a few PTGR1 inhibitors have been reported and none has entered clinical trials to date. All of them suffer from low activity with $\mathrm{IC}_{50}$ values at $\mu \mathrm{M}$ range. High-throughput screening, structure-based drug design, and virtual screening should be used to identify novel and potent PTGR1 modulators. These modulators will serve as chemical tools to further investigate the function of PTGR1 and drug leads for future cancer treatment. Interestingly, PTGR1 has also been linked to not only HMAF and other prodrug sensitivity, but also cancer oxidative stress. These results suggest that an PTGR1 activator might benefit HMAF anti-cancer treatment and an PTGR1 inhibitor might sensitize cancer cells to ROS-induced cell death. Therefore, combinational therapy approaches appear to be an interesting research direction for the utilization of PTGR1 modulators.

\section{AUTHOR CONTRIBUTIONS}

XW, GY, WZ, KS, LZ, and ZG collected the literature and wrote the article. $Z G$ edited and made significant revisions to the article. All authors read and approved the final.

\section{ACKNOWLEDGMENTS}

This work was made possible by the research foundation of talented scholars from Chongqing Medical University. The authors thank Dr. Junyan Liu and Marnie Kotlyar for critical comments on the article.

Gong, J., Neels, J. F., Yu, X., Kensler, T. W., Peterson, L. A., and Sturla, S. J. (2006). Investigating the Role of Stereochemistry in the Activity of Anticancer Acylfulvenes: Synthesis, Reductase-Mediated Bioactivation, and Cellular Toxicity. J. Med. Chem. 49, 2593-2599. doi:10.1021/jm051104t

Győrffy, B., Surowiak, P., Budczies, J., and Lánczky, A. (2013). Online Survival Analysis Software to Assess the Prognostic Value of Biomarkers Using Transcriptomic Data in Non-Small-Cell Lung Cancer. PLoS One 8, e82241e82241. doi:10.1371/journal.pone.0082241

Hanahan, D., and Weinberg, R. A. (2011). Hallmarks of Cancer: The Next Generation. Cell 144, 646-674. doi:10.1016/j.cell.2011.02.013

Hatem, E., Azzi, S., El Banna, N., He, T., Heneman-Masurel, A., Vernis, L., et al. (2019). Auranofin/Vitamin C: A Novel Drug Combination Targeting TripleNegative Breast Cancer. J. Natl. Cancer Inst. 111, 597-608. doi:10.1093/jnci/ djy149

Hiam-Galvez, K. J., Allen, B. M., and Spitzer, M. H. (2021). Systemic Immunity in Cancer. Nat. Rev. Cancer 21, 345-359. doi:10.1038/s41568-021-00347-z

Hori, T., Ishijima, J., Yokomizo, T., Ago, H., Shimizu, T., and Miyano, M. (2006). Crystal Structure of Anti-Configuration of Indomethacin and Leukotriene B4 
12-Hydroxydehydrogenase/15-Oxo-Prostaglandin 13-reductase Complex Reveals the Structural Basis of Broad Spectrum Indomethacin Efficacy. J. Biochem. 140, 457-466. doi:10.1093/jb/mvj176

Itoh, K., Yamamoto, K., Adachi, M., Kosaka, T., and Tanaka, Y. (2008). Leukotriene B412-Hydroxydehydrogenase/15-Ketoprostaglandin $\delta 13-$ Reductase (LTB412-HD/PGR) Responsible for the Reduction of a DoubleBond of Thea, $\beta$-Unsaturated Ketone of an Aryl Propionic Acid Non-steroidal Anti-inflammatory Agent CS-670. Xenobiotica 38, 249-263. doi:10.1080/ 00498250701767667

Jeon, W.-K., Choi, J., Park, S. J., Jo, E. J., Lee, Y. K., Lim, S., et al. (2015). The Proinflammatory LTB4/BLT1 Signal axis Confers Resistance to TGF-B1Induced Growth Inhibition by Targeting Smad3 Linker Region. Oncotarget 6, 41650-41666. doi:10.18632/oncotarget.6146

Laks, D. R., Crisman, T. J., Shih, M. Y. S., Mottahedeh, J., Gao, F., Sperry, J., et al. (2016). Large-Scale Assessment of the Gliomasphere Model System. Neuonc. 18, 1367-1378. doi:10.1093/neuonc/now045

Liu, M., Liu, Y., Deng, L., Wang, D., He, X., Zhou, L., et al. (2018). Transcriptional Profiles of Different States of Cancer Stem Cells in Triple-Negative Breast Cancer. Mol. Cancer 17, 65. doi:10.1186/s12943-018-0809-x

Loud, J. T., and Murphy, J. (2017). Cancer Screening and Early Detection in the 21 St Century. Semin. Oncol. Nurs. 33, 121-128. doi:10.1016/j.soncn.2017.02.002

McMorris, T. C., Elayadi, A. N., Yu, J., Hu, Y., and Kelner, M. J. (1999). Metabolism of Antitumor Hydroxymethylacylfulvene by Rat Liver Cytosol. Drug Metab. Dispos. 27, 983-985.

Mesa, J., Alsina, C., Oppermann, U., Parés, X., Farrés, J., and Porté, S. (2015). Human Prostaglandin Reductase 1 (PGR1): Substrate Specificity, Inhibitor Analysis and Site-Directed Mutagenesis. Chemico-Biological Interactions 234, 105-113. doi:10.1016/j.cbi.2015.01.021

Oh, D.-Y., and Bang, Y.-J. (2020). HER2-targeted Therapies - A Role beyond Breast Cancer. Nat. Rev. Clin. Oncol. 17, 33-48. doi:10.1038/s41571-019-0268-3

Primiano, T., Gastel, J. A., Kensler, T. W., and Sutter, T. R. (1996). Isolation of cDNAs Representing Dithiolethione-Responsive Genes. Carcinogenesis 17, 2297-2303. doi:10.1093/carcin/17.11.2297

Raymond, E., Kahatt, C., Rigolet, M. H., Sutherland, W., Lokiec, F., Alexandre, J., et al. (2004). Characterization and Multiparameter Analysis of Visual Adverse Events in Irofulven Single-Agent Phase I and II Trials. Clin. Cancer Res. 10, 7566-7574. doi:10.1158/1078-0432.ccr-04-0869

Roberts, L. S., Yan, P., Bateman, L. A., and Nomura, D. K. (2017). Mapping Novel Metabolic Nodes Targeted by Anti-Cancer Drugs that Impair Triple-Negative Breast Cancer Pathogenicity. ACS Chem. Biol. 12, 1133-1140. doi:10.1021/ acschembio.6b01159

Salas-Benito, D., Pérez-Gracia, L. J., Ponz-Sarvisé, M., Rodriguez-Ruiz, E. M., Martínez-Forero, I., Castañón, E., et al. (2021). Paradigms on Immunotherapy Combinations with Chemotherapy. Cancer Discov. 11, 1353-1367. doi:10.1158/ 2159-8290.CD-20-1312

Sánchez-Rodríguez, R., Torres-Mena, J. E., Quintanar-Jurado, V., Chagoya-Hazas, V., Rojas del Castillo, E., del Pozo Yauner, L., et al. (2017). Ptgr1 Expression Is Regulated by NRF2 in Rat Hepatocarcinogenesis and Promotes Cell Proliferation and Resistance to Oxidative Stress. Free Radic. Biol. Med. 102, 87-99. doi:10.1016/j.freeradbiomed.2016.11.027

Siegel, R. L., Miller, K. D., Fuchs, H. E., and Jemal, A. (2021). Cancer Statistics, 2021. CA A. Cancer J. Clin.Cancer J. Clin. 71, 7-33. doi:10.3322/caac.21654

Sokolenko, A. P., and Imyanitov, E. N. (2018). Molecular Diagnostics in Clinical Oncology. Front. Mol. Biosci. 5, 76. doi:10.3389/fmolb.2018.00076

Sung, H., Ferlay, J., Siegel, L. R., Laversanne, M., Soerjomataram, I., Jemal, A., et al. (2021). Global Cancer Statistics 2020: GLOBOCAN Estimates of Incidence and
Mortality Worldwide for 36 Cancers in 185 Countries. CA. Cancer J. Clin. 71, 209-249. doi:10.3322/caac.21660

Tapak, L., Saidijam, M., Sadeghifar, M., Poorolajal, J., and Mahjub, H. (2015). Competing Risks Data Analysis with High-Dimensional Covariates: An Application in Bladder Cancer. Genomics, Proteomics \& Bioinformatics 13, 169-176. doi:10.1016/j.gpb.2015.04.001

Vitturi, D. A., Chen, C.-S., Woodcock, S. R., Salvatore, S. R., Bonacci, G., Koenitzer, J. R., et al. (2013). Modulation of Nitro-Fatty Acid Signaling. J. Biol. Chem. 288, 25626-25637. doi:10.1074/jbc.m113.486282

Wang, B., Wu, L., Chen, J., Dong, L., Chen, C., Wen, Z., et al. (2021). Metabolism Pathways of Arachidonic Acids: Mechanisms and Potential Therapeutic Targets. Signal. Transduct. Target. Ther. 6, 1-30. doi:10.1038/s41392-020-00443-w

Wang, D., and Dubois, R. N. (2010). Eicosanoids and Cancer. Nat. Rev. Cancer 10, 181-193. doi:10.1038/nrc2809

Xue, L., Zhu, Z., Wang, Z., Li, H., Zhang, P., Wang, Z., et al. (2016). Knockdown of Prostaglandin Reductase 1 (PTGR1) Suppresses Prostate Cancer Cell Proliferation by Inducing Cell Cycle Arrest and Apoptosis. Biosci. Trends 10, 133-139. doi:10.5582/bst.2016.01045

Yang, W. J., Wang, H. B., Wang, W. D., Bai, P. Y., Lu, H. X., Sun, C. H., et al. (2020). A Network-Based Predictive Gene Expression Signature for Recurrence Risks in Stage II Colorectal Cancer. Cancer Med. 9, 179-193. doi:10.1002/cam4.2642

Yokomizo, T., Izumi, T., Takahashi, T., Kasama, T., Kobayashi, Y., Sato, F., et al. (1993). Enzymatic Inactivation of Leukotriene B4 by a Novel Enzyme Found in the Porcine Kidney. Purification and Properties of Leukotriene B4 12-hydroxydehydrogenase. J. Biol. Chem. 268, 18128-18135. doi:10.1016/s0021-9258(17)46820-7

Yu, X., Erzinger, M. M., Pietsch, K. E., Cervoni-Curet, F. N., Whang, J., Niederhuber, J., et al. (2012). Up-Regulation of Human Prostaglandin Reductase 1 Improves the Efficacy of Hydroxymethylacylfulvene, an Antitumor Chemotherapeutic Agent. J. Pharmacol. Exp. Ther. 343, 426-433. doi:10.1124/jpet.112.195768

Yue, W. W., Shafqat, N., Krojer, T., Pike, A. C. W., von Delft, F., Sethi, R., et al. (2011). RCSB PDB - 2Y05: Crystal Structure of Human Leukotriene B4 12hydroxydehydrogenase in Complex with NADP and Raloxifene. Available at: https://www.rcsb.org/structure/2y05 (Accessed May 30, 2021). doi:10.2210/ pdb2y05/pdb

Zhao, Y., Weng, C.-C., Tong, M., Wei, J., and Tai, H.-H. (2010). Restoration of Leukotriene B4-12-Hydroxydehydrogenase/15- Oxo-Prostaglandin 13reductase (LTBDH/PGR) Expression Inhibits Lung Cancer Growth In Vitro and In Vivo. Lung Cancer 68, 161-169. doi:10.1016/j.lungcan.2009.06.011

Conflict of Interest: The authors declare that the research was conducted in the absence of any commercial or financial relationships that could be construed as a potential conflict of interest.

Publisher's Note: All claims expressed in this article are solely those of the authors and do not necessarily represent those of their affiliated organizations, or those of the publisher, the editors and the reviewers. Any product that may be evaluated in this article, or claim that may be made by its manufacturer, is not guaranteed or endorsed by the publisher.

Copyright $\odot 2021$ Wang, Yin, Zhang, Song, Zhang and Guo. This is an open-access article distributed under the terms of the Creative Commons Attribution License (CC $B Y$ ). The use, distribution or reproduction in other forums is permitted, provided the original author(s) and the copyright owner(s) are credited and that the original publication in this journal is cited, in accordance with accepted academic practice. No use, distribution or reproduction is permitted which does not comply with these terms. 